\title{
Algunas consideraciones en torno al software para Educación Infantil
}

\section{Santos Urbina}

santos.urbina@uib.es

En los últimos años, la industria del software en nuestro país está centrando muchos de sus esfuerzos en el sector de edades que comprende la etapa de Educación Infantil ${ }^{1}$. Bajo la vieja fórmula de "aprender jugando", y exprimiendo al máximo las cualidades multimedia de los actuales equipos informáticos, se están ofertando atractivos programas de impecable factura.

Y a juzgar por el crecimiento a que aludíamos, no cabe duda que se trata de un negocio en alza. Un estudio estadounidense realizado hace apenas cuatro años revelaba que el $67 \%$ del software allí publicado tenía como destinatarios a los niños pequeños (Haugland, 1998).

Así, actualmente podemos adquirir programas educativos cuyos destinatarios tienen incluso menos de tres años. No es ya una excepción encontrar algún programa dirigido a niños de 18 meses $^{2}$. Al mismo tiempo, podemos observar

\footnotetext{
${ }^{1}$ Conviene aclarar a los lectores no españoles que la etapa de Educación Infantil se divide en España en dos ciclos: de 0 a 3 años, y de 3 a 6 años.

${ }^{2}$ En el momento de escribir estas líneas (octubre 2000), al menos existen en nuestro país tres compañías que disponen de algún producto destinado a niños de un año y medio, en concreto, Anaya, Fisher Price y The Learning Company.
} 
que compañías tan poderosas como Microsoft han sacado al mercado periféricos específicos destinados a niños de entre 2 y 6 años ${ }^{3}$.

Asimismo, prácticamente todas las grandes editoriales de nuestro país se han ido sumando a esta tendencia y ya cuentan con un departamento de productos multimedia o similar. Y, obviamente, la confluencia de destinatarios también ha propiciado que el sector juguetero esté invirtiendo sus esfuerzos en este ámbito.

Otro de los indicadores que nos muestran el crecimiento de la industria dirigida a este sector es la aparición cada vez más frecuente de artículos alusivos al tema en revistas de informática de consumo.

Sin entrar en la polémica acerca de si el interés comercial se deriva del interés social o el primero, a través de campañas publicitarias, genera el segundo, lo cierto es que la presencia de una informática centrada en los niños pequeños se está haciendo progresivamente patente.

Además de la creciente oferta, una buena parte de los productos comerciales en el mercado dirigidos a las edades de referencia suelen tener unas características muy heterogéneas o son traducciones del inglés (u otros idiomas) sin una adaptación a las características de nuestra población; otros se limitan a "trasplantar" algún personaje popular entre los niños desde la televisión o el cine a la pantalla del ordenador; y, por supuesto, algunos de ellos están realizados con gran calidad.

El interrogante que surge de esta situación es claro: ¿poseen todos estos productos la calidad necesaria para considerarse "educativos"? Claro está que una concepción amplia del calificativo "educativo" nos llevaría a considerar como tales a la práctica totalidad de programas existentes en el mercado. Cabría, entonces, plantearse otras formas más restrictivas para determinar la idoneidad de los materiales informáticos dirigidos a la Educación Infantil. Soy consciente, de acuerdo con Haugland (1992), de que los efectos del ordenador en "niños preescolares" -como en otras etapas educativas- dependerán de

\footnotetext{
${ }^{3}$ Se trata del producto Easyball, similar a los trackballs (especie de ratón invertido) cuya bola es mucho mayor y de mucho más fácil control.

http://www.windows.com/spain/home/kids/easyball.htm
} 
cómo sea utilizado, y será responsabilidad de los adultos realizar las elecciones apropiadas para que resulte beneficioso. Autores como Yager y otros (1993) abundan en esta idea, remarcando la importancia en la selección de un software adecuado. Tanto uno como otros coinciden en la importancia de una utilización del ordenador contextualizada en el aula, de tal forma que suponga una herramienta más. Por su parte, Shade (1996) pone de manifiesto el papel fundamental de los maestros para que los supuestos "beneficios" de la tecnología puedan llevarse a cabo; así, la decisión más crítica que el educador deberá adoptar se refiere a la elección del software, ya que, en palabras del autor, los ordenadores no son, en definitiva, más que "plástico y circuitos" que no alcanzan su potencial hasta que se cargan los programas.

Un estudio desarrollado en Estados Unidos por Haugland y Shade (1994) hace unos años demostraba que tan sólo un $25 \%$ de los programas educativos dirigidos a un público "preescolar" estaban desarrollados adecuadamente ${ }^{4}$.

La razón de ello parece más o menos clara: sencillamente se trata de productos dirigidos a un sector de edad determinado que han sido ideados y elaborados con una finalidad casi exclusivamente lucrativa.

Ignoro si pasados más de cinco años tras el estudio de estos autores los datos se han visto modificados para mejor o no, aunque sí me gustaría ser algo más optimista -aunque con moderación y aún sin disponer de estudios similarescon respecto a la calidad de los materiales producidos y distribuidos en el estado español.

Al hilo de lo dicho, quisiera aclarar que la mayoría de ejemplos que intentan ilustrar el discurso en las páginas que siguen se corresponden a casos reales, si bien no he creído oportuno ofrecer las referencias de ninguno de ellos porque no es mi objetivo el denostar o menospreciar trabajo alguno -o alabar, en otras ocasiones-, sino tan solo exponer de manera más clara mis reflexiones.

\footnotetext{
${ }^{4}$ Y ello considerando que había en Estados Unidos, según datos de los autores Haugland y Shade 1994), un total de 144 empresas dedicadas a esta industria, datos tremendamente alejados de la realidad actual de nuestro país.
} 
A continuación quisiera, pues, exponer algunas consideraciones acerca del software dirigido a los niños de Educación Infantil. No pretendo ser exhaustivo -bien seguro que quedan muchas cosas sobre las que hablar-, ni he seguido un orden riguroso a la hora de abordar los diferentes temas. Se trata más bien de "pinceladas" breves, sobre las cuales posiblemente cabría un análisis mucho más profundo y detenido de lo que la extensión de un artículo permite.

Podría aducirse que algunos de estos temas no atañen exclusivamente a los materiales para los más pequeños, cosa del todo cierta, aunque precisamente por ser más generales no dejan de influir en ellos. Por otra parte, anticipar que la mayoría de los tópicos tratados están relacionados de una forma u otra con el diseño o la planificación de los materiales, si bien también hay cabida para otros más anecdóticos.

\section{- La presentación del producto}

Frases pretendidamente impactantes. El software educativo no escapa a los dictados de la publicidad. Las empresas que se dedican a este negocio no pueden lanzar al mercado productos que no generen beneficios. Será, por lo tanto, imprescindible asegurar un mínimo de ventas. Como muy bien se ha estudiado en el ámbito de los videojuegos (Provenzo, 1991) una presentación llamativa, con ilustraciones vistosas y frases impactantes puede ser un buen reclamo. Y el ámbito de lo educativo no ha escapado a estos dictados. Así, no es raro encontrar programas en cuyas cajas figuran frases del tipo: "Sonido digitalizado!!" o "CD ROM interactivo" centrarnos en estos dos ejemplos, a nadie ligeramente conocedor del tema se le escapa que el sonido que se ofrezca en un CD ROM habrá sido fforzosamente digitalizado!; y, por otra parte, sería, más que conveniente, obligado que un programa educativo ofrecido en soporte informático fuese interactivo...!

El regalo adjunto. Los vendedores saben perfectamente que un buen estímulo al consumo es regalar un producto adicional a los clientes con la

\footnotetext{
${ }^{5}$ Las frases expuestas, lejos de ser suposiciones, son reales y literales.
} 
compra de algún artículo. Así, cada vez es más frecuente que la caja de software incluya una figurita del personaje central, un póster o un estuche de lápices de colores añadiendo de forma muy clara -eso sí-, los epítetos "gratis" o "regalo". En aquellos casos en los que se ofrece una propuesta didáctica complementaria -al software- es posible justificar la inclusión de alguno de esos u otros presentes desde un punto de vista educativo; sin embargo, suelen responder a la estrategia de ventas centrada en el reclamo, más que a ningún otro tipo de consideración.

Además, en mi opinión, algunos de los productos que adjuntan este tipo de obsequios tienen la suficiente calidad como para hacer valer sus cualidades sin reclamo alguno.

\section{- Dispersión de edades/ inespecificidad del destinatario}

De acuerdo con algunos autores (Shade, 1996), un buen programa educativo debería ser adaptable a diferentes niveles de desarrollo. Sin embargo, no ha de confundirse esa adaptabilidad con las etiquetas que aparecen en determinados productos anunciando su utilización por amplios abanicos de edades. Por poner un ejemplo, hay programas que indican en el envoltorio: "de 2 a 8 años". En algunos casos, ese tipo de software camufla cócteles de juegos o ejercicios donde lo más probable es que no se haya diseñado ninguno de ellos para una edad determinada $\mathrm{y}$, donde, obviamente, sólo algunos podrán ser resueltos por los más pequeños, mientras que los niños de mayor edad se aburrirán prácticamente con la totalidad de propuestas. Otra cuestión adicional se refiere a aspectos relacionados con el diseño de la aplicación desde una perspectiva general: las ilustraciones, la interfase, la estructura del programa, el tipo de vocabulario empleado, etc., ¿se verán modificados en función de la edad de quien utilice el programa? A tenor de lo visto, difícilmente puede conseguirse algo así cuando el abanico de edades es tan amplio.

\section{- Instrucciones/ consignas/ indicaciones}


En ocasiones las consignas se ofrecen de forma exclusivamente verbal ya que los programas se dirigen a un público no lector. Sin embargo, dada la edad de los destinatarios, es preciso que sean sumamente claras por lo que se refiere al lenguaje empleado y completas, en tanto no dejen dudas sobre qué se ha de hacer.

Por supuesto, otro aspecto a cuidar, redundando en lo anterior, es la calidad técnica del sonido -factor de gran importancia. Parámetros como el volumen de grabación y la "limpieza" del sonido deben tenerse en cuenta de manera muy especial. Las explicaciones que se ofrecen al usuario deben ser claramente audibles e inteligibles. A título anecdótico decir, que hace algún tiempo apareció un programa destinado al público infantil entre cuyas virtudes destacaba el estar adaptado para niños con problemas de audición; el único inconveniente radicaba en que los elementos de audio precisamente-, eran de pésima calidad.

\section{- Menús/ barras de botones}

En primer lugar, decir que, como parte fundamental del sistema de comunicación con el usuario, convendría que fuesen estables en cuanto a su ubicación y diseño. Si esto es necesario cuando se trata de diseñar un programa dirigido a público adulto (Price, 1991; Cacheiro, 1997), tanto más lo será cuando los destinatarios son niños pequeños. Por otra parte, los iconos deberían ser lo suficientemente claros para identificar su utilidad con relativa facilidad. Según Abramson (1998), también deberían ser de un tamaño lo suficientemente grande y separados entre sí para evitar clics involuntarios.

También resulta interesante que ofrezcan "pistas" luminosas o sonoras cuando hayan de ser activados, como un ligero destello o parpadeo, lo que sugerirá al niño la conveniencia de mover el puntero hacia ese lugar. Sin embargo, considero que los efectos visuales espectaculares pueden desviar la atención del sujeto de su objetivo inicial (no hay que perder de vista que los niños pequeños suelen tener una atención bastante lábil), por lo que habría que ser comedidos en la aplicación de los mismos. 
Es posible que el programa tenga muchas opciones de configuración. Convendría que cada una de esas opciones no supusiera un botón adicional, lo que aumentaría la complejidad de funcionamiento del programa y por añadidura la dificultad para manejarlo con cierto nivel de autonomía.

Dependiendo del programa es posible que dichas opciones estén dirigidas a los adultos, quienes serán los encargados de configurar los parámetros del programa. Sería, pues, preferible que se pudiera acceder a ellas mediante una combinación de teclas, para evitar su activación accidental en el transcurso del juego.

Por otra parte, un exceso de botones pueden actuar como distractores de la tarea y también hacer que se salga del programa de forma involuntaria debido, por ejemplo, a la curiosidad o a la falta de destreza.

\section{- Los punteros}

Totalmente ligados al diseño de la aplicación, los punteros tienen una vital importancia dado que son normalmente el elemento móvil en pantalla mediante el cual se interactúa con el programa. Así pues, será preciso que cumplan una serie de características básicas según los destinatarios a que nos estamos refiriendo:

- deberán ser lo suficientemente grandes para ser visibles en todo momento sin dificultad, pero no tanto como para impedir ver otros elementos importantes que aparezcan en pantalla al superponerse a ellos;

- totalmente relacionado con el punto anterior, el color debería ser lo suficientemente contrastado con el fondo en todo momento;

- los punteros móviles y/o efectistas (como por ejemplo, una varita mágica chispeante) pueden ser espectaculares y muy llamativos pero han de garantizar no despistar al sujeto de la actividad central y, volviendo a lo anterior, estar perfectamente localizables;

- en mi opinión, puede ser interesante que adopten diferentes formas en función de lo que el sujeto esté haciendo (por ejemplo, si 
recurrimos al popular puntero en forma de mano, este podrá ser un dedo señalador, una mano abierta, o asiendo algo, etc.) ya que pueden ser un elemento contextualizador más; ahora bien, deberían de pasar lo más desapercibidos posible de manera que no interfiriesen en la tarea que se está llevando a cabo.

\section{- Uso tutelado o autónomo}

Una determinación importante para los diseñadores del programa será concretar hasta qué punto se pretende la intervención autónoma del sujeto. No sólo los presupuestos teóricos de partida o los objetivos pretendidos, sino también la complejidad del programa, deberían apuntar hacia un tipo de diseño más centrado en la tutela o en la utilización autónoma ${ }^{6}$.

Personalmente me decanto más por la segunda opción, si bien entiendo que dadas las características de los sujetos en algunos casos la mera elección de una opción puede resultar complicado para ellos. Quisiera insistir, pues, en la necesidad de establecer un equilibrio entre dotar al programa de múltiples opciones de configuración y simplificar al máximo su utilización para permitir a los niños un uso lo más autónomo posible.

\section{- Complejidad cognitiva de las tareas}

Aquellos programas que se componen de diversas propuestas basadas en un combinado de actividades deberían no perder de vista que los niveles de dificultad deberían ser similares de una a otra. Así, por ejemplo, el típico juego de memoria consistente en asociar parejas de imágenes inicialmente vueltas hacia abajo, aparece en un paquete de software infantil junto con otras actividades; destaca entre ellas una versión de este mismo juego, pero basado en sonidos -en lugar de imágenes. Aumenta tan rápidamente la dificultad que incluso un adulto tiene serios problemas para resolverlo.

\footnotetext{
${ }^{6}$ Obviamente, la edad a que se dirige el mismo implicará de por sí ciertas limitaciones o posibilidades. Así, los materiales dirigidos a los más pequeños (recordemos que hay materiales para niños de 18 meses), requieren forzosamente la concurrencia del adulto por cuestiones evidentes.
} 
En el caso de que las propuestas de actividades estuvieran secuenciadas, incrementándose la dificultad a medida que se avanza, el programa debería cuidar especialmente que no se produjesen grandes desfases de un nivel a otro.

Actualmente podemos encontrar en el mercado algunos programas que presentan propuestas muy correctas, conscientes de estos inconvenientes. Así, las diferentes actividades constan de niveles de dificultad diferentes $y$, adicionalmente, el usuario -el educador, en este caso- puede ser orientado al respecto.

Materiales algo más complejos -basados en inteligencia artificial- pueden adaptarse a las respuestas que el sujeto va ofreciendo, modificando de manera automática sus propuestas para adaptarse al ritmo y nivel detectados. Como apunta Hohmann (1998), el buen software facilita un adecuado nivel de entrada al sujeto y, de la misma manera, se va adecuando al progreso del sujeto.

\section{- Adaptación a las capacidades psicomotoras de los niños}

El proceso de maduración continuada de los niños hace que en ocasiones no hayan conseguido las destrezas mínimas para conseguir realizar adecuadamente una propuesta, pese a comprender correctamente las instrucciones y saber perfectamente qué han de hacer. Un ejemplo muy claro lo encontramos en el tamaño de los objetos presentados en pantalla sobre los que se ha de hacer clic; un ejercicio específico puede solicitar al niño que resuelva una determinada tarea y haga clic al finalizarla sobre un botón que, desgraciadamente, es tan pequeño que resulta más difícil acertar sobre él que resolver el problema propuesto. Hay que tener en cuenta que una tarea tan simple para un adulto como hacer clic sobre un determinado punto de la pantalla (que es totalmente automática) puede albergar serias dificultades para un niño de tres años que deberá controlar: el movimiento de su mano, el desplazamiento del puntero en la pantalla, su inmovilidad sobre el lugar deseado y, añadido a esto, conseguir hacer clic sin que se mueva el ratón. 
Sería en estos casos conveniente que se comprobase previamente hasta qué punto los niños pueden utilizar de forma adecuada el programa de la manera propuesta y ofrecer las alternativas pertinentes (en el ejemplo, diseñar botones de mayor tamaño, o bien permitir que el botón en cuestión se active simplemente al detectar el desplazamiento del puntero en lugar del clic, etc.)

\section{- Tratamiento de los errores}

Los presupuestos teóricos de que parte el productor de software determinan la manera en que entiende cómo se produce el aprendizaje y, por ende, estas concepciones se reflejarán en los materiales realizados (Urbina, 1999). Así, el tratamiento del error puede ser considerado de forma muy diferente si partimos de una concepción conductista o constructivista, por citar la situación más típica. Sin entrar a debatir acerca de las bondades o inconvenientes de estas opciones sí quisiera hacer una observación acerca del tratamiento del error que se realiza en algunos programas.

Por lo general, el atractivo que desprende para los más pequeños el ordenador es motivo más que suficiente para que quieran sentarse ante la pantalla y ver qué está pasando ahí dentro. Poder interactuar con personajes de ficción, conseguir llevar a cabo determinadas propuestas y que estas sean aplaudidas, supone un valor añadido para que muestren su entusiasmo. Sin embargo, hay que tener en cuenta que los niños pequeños también tienen un umbral de frustración mucho menor que los adultos. En definitiva, ante el ordenador buscan pasarlo bien. Que un determinado programa conteste de forma tajante: “iMal!. ¡Lo has hecho mal!”, cuando se desvían de las respuestas previstas, y la misma o similar indicación es reiterada en nuevos intentos, sólo lleva a que el sujeto desvíe su atención más allá de la pantalla, se levante y cambie de actividad. El objetivo de los programadores, sin duda bienintencionado, consiste en que no se repita la respuesta no deseada; posiblemente lo consigan, pero también corren el riesgo de que no se repita ninguna respuesta más. 
En los tipos de programa en que se valora el resultado sancionando la respuesta que da el sujeto se debería intentar utilizar fórmulas más "suaves" al producirse el error ${ }^{7} \mathrm{y}$, muy importante, orientarlo hacia la respuesta esperada. Según Abramson (1998), tras dos intentos infructuosos el programa debería ofrecer la respuesta correcta sin obligar al sujeto a repetir el mismo proceso.

\section{- Variables dialectales o idiomáticas}

La capacidad de almacenamiento del CD-ROM hace posible, en numerosas ocasiones, que se ofrezca al usuario la posibilidad de seleccionar el idioma en que va a utilizar el programa.

Esto supone una gran ventaja en comunidades bilingües 0 , desde un punto de vista comercial, la posibilidad de lanzar un mismo producto en diferentes contextos idiomáticos reduciendo costes.

El problema asociado se deriva de la estandarización lingüística -o precisamente de su ausencia ${ }^{8}$. Obviamente, existen múltiples variantes idiomáticas entre el español hablado en México, en Argentina, en Cuba o en España que, fundamentalmente, afectan al vocabulario y a expresiones, giros lingüísticos o frases hechas, amén de la pronunciación y la cadencia, lo que podríamos denominar como el "acento" (bien es cierto, que incluso dentro de un mismo país se pueden producir circunstancias similares aunque de una envergadura mucho menor).

Así, no es raro encontrar en el mercado español productos manufacturados en Estados Unidos que, efectivamente, ofrecen la posibilidad de selección de nuestro idioma, pero cuya variante diferirá notablemente del estándar aquí hablado.

\footnotetext{
${ }^{7}$ Tampoco se trata, como bien apuntan Dell y Newton (1998) de que el estímulo que preceda al error sea tan festivo que pueda producir confusión en el sujeto acerca de lo adecuado de su respuesta.

${ }^{8}$ Como apunta Marqués $(1995,182)$, otro problema asociado de no menor importancia, estribará en la "cultura y entorno". Las situaciones presentadas en los programas pueden diferir notablemente de un país a otro, lo que puede confundir más a los más pequeños dado su escaso bagaje experiencial.
} 
No es que se trate de un aspecto de vital importancia, dado que incluso puede ser enriquecedor que los niños conozcan otras variantes del idioma en que se comunican. Sin embargo, dado que hablamos de niños de corta edad, sería más que conveniente tenerlo en cuenta.

Si nos referimos al estado español, algo similar ocurre con aquellos programas realizados, por ejemplo, en la comunidad catalana que se ofertan en las otras comunidades catalanohablantes. Las diferencias entre el catalán que se habla, por ejemplo, en Barcelona y el de Palma de Mallorca pueden centrarse -simplificando- en la entonación, la pronunciación de determinadas vocales, algo de vocabulario y algunas terminaciones verbales. Estas diferencias pueden no suponer un gran problema y, por supuesto, no ser un impedimento para la comunicación, pero pueden crear cierta confusión en un niño pequeño ${ }^{9}$.

\section{- Guías didácticas}

En mi opinión es este un elemento de inclusión obligada en todo material con intencionalidad educativa manifiesta. Ya sea en versión impresa o electrónica, debería adjuntarse una guía detallada con orientaciones para educadores y padres, donde se expliciten los objetivos pretendidos, se sugieran modalidades de utilización del material, etc. Obviamente, sin pretender que se incluyan "manuales de uso" en cuanto a volumen -no creo que sea el sentido pretendido-, estas guías deberían ser materiales suficientemente elaborados. En el mercado podemos encontrar diferentes modalidades de material por lo que a este tema respecta: aquellos que incluyen únicamente algunas frases en la portada; los que adjuntan tan sólo una hoja explicativa -la cual, por otra parte, no suele ofrecer mucha más información que los primeros; los que incluyen abundante información pero referida casi exclusivamente al funcionamiento y características

\footnotetext{
${ }^{9}$ Como muestra podemos ver algunas diferencias entre vocablos de uso común en el catalán de Cataluña y el de Baleares (por este orden): abuelo, avi - padrí; agua, aigua - aigo; vaso, got - tassó; tomate, tomàquet - tomàtiga; lechuga, ensiam - lletuga; jamón, pernil - cuixot; perro, gos - ca; gato, gat - moix; pantalones, pantalons - calçons; calcetines, mitjons - calcetins.
} 
técnicas del programa $^{10} ;$ y aquellos que incluyen auténticas guías didácticas. Es de destacar que afortunadamente cada vez son más frecuentes estos últimos.

La inclusión de una buena guía didáctica debería ser garantía de la presencia de algún profesional de la educación en el equipo de producción del material, lo cual no sólo es deseable, sino necesario -aunque las empresas no siempre lo contemplen de la misma forma.

Para terminar quisiera exponer una idea que, como poco, me parece interesante. Al margen de su intención final, o si están pensados desde una perspectiva más económica que pedagógica, lo cierto es que la inmensa mayoría de materiales son utilizados con fines educativos, sea en contextos formales o no. De hecho, uno de los reclamos publicitarios que mayor efecto parece surtir entre los consumidores de este tipo de productos, consiste en poner de forma visible en algún lugar de la caja la etiqueta "educativo". Si ello les permite llegar a un mayor número de público, objetivo conseguido. Ahora bien, la utilización del término "educativo" puede responder, efectivamente, a una descripción precisa del producto o puede también camuflar materiales de ínfima calidad. No quisiera pecar de corporativismo y dar la impresión de que determinado vocabulario -máxime tratándose de términos de uso común, no incluidos en la jerga ininteligible del colectivo- sólo puede ser empleado por los profesionales de la educación; lo que sí considero es que no debería engañarse al público ofreciendo como educativos productos que no lo son.

¿Sería, pues, absurda la idea de que este tipo de software pasase por controles de calidad -por supuesto, externos a los productores- desde el punto de vista educativo que garantizasen las cualidades del producto? Creo que convendría reflexionar sobre ello...

\footnotetext{
${ }^{10}$ Aspecto este muy a tener en cuenta también: si el material precisa de una amplia guía de uso por lo que respecta a sus características técnicas tal vez estemos ante un programa demasiado complejo que no permita su manejo a los niños con un mínimo de autonomía.
} 


\section{REFERENCIAS}

Abramson, G. (1998) How to evaluate educational software. Principal (Reston, Va.), $v$ 78, 1, 60-61.

Cacheiro, M.L. (1997) El interfaz de navegación. Diseño pedagógico. En J. Gallego y C.M. Alonso (eds.) Multimedia (pp. 113-133). Madrid: UNED.

Dell, A.G. y Newton, D. (1998) Software for play and active early learning. Exceptional Parent, nov. 1998, 39-43.

Haugland, S.W. (1992) The effect of Computer Software on Preschool Children's Developmental Gains. Journal of Computing in Childhood Education, 3, 15-30.

Haugland, S.W. y Shade, D.D. (1994) Software Evaluation for Young Children. En J.L. Wright y D.D. Shade (eds.), Young Children: Active Learners in a Technological Age. Washington: NAEYC.

Haugland, S.W. (1998) The best developmental software for young children. Early Childhood Education Journal, v. 25, 4, 247-254.

Hohmann, C. (1998) Evaluating and selecting software for children. Child Care Information Exchange, 9, 60-63.

Marqués, P. (1995) Software educativo. Guía de uso y metodología de diseño. Barcelona: Estel.

Price, R.V. (1991) Computer-aided instruction. A Guide for Authors. Pacific Grove, California: Brooks/ Cole Publishing Company.

Provenzo, E.F. (1991) Video kids: making sense of Nintendo. Cambridge: Harvard University Press.

Shade, D.D. (1996) Software evaluation. Young children, 5 (6), 17-21.

Urbina, S. (1999) Informática y teorías del aprendizaje. Pixel Bit. Revista de Medios y Comunicación, 12, 87-100.

Yager, R.E., Blunck, S.M. y Nelson, E.T. (1993) The Use of Computers to Enhance Science Instruction in Pre-School and K-3 Classrooms. Journal of Computing in Childhood Education, 4(2), 125-136. 
Santos Urbina

Dpt. Ciencias de la Educación

Universitat de les Illes Balears

España

santos.urbina@uib.es

http://www.uib.es/depart/gte/personal/sur 